\title{
Reabilitação fonoaudiológica na paralisia facial periférica: revisão integrativa
}

\author{
Speech-language rehabilitation in peripheral facial paralysis: \\ integrative review
}

\author{
Marlos Passos Dias ${ }^{1}$ (D), Mabile Francine Ferreira Silva² (D), Simone dos Santos Barreto ${ }^{3}$ (D)
}

\begin{abstract}
RESUMO
Objetivos: identificar e analisar a produção científica sobre as estratégias terapêuticas empregadas na reabilitação fonoaudiológica de pessoas com paralisia facial periférica (PFP). Estratégia de pesquisa: foi realizada uma revisão integrativa, utilizando-se as bases de dados SciELO, PubMed, Web of Science, ScienceDirect e Portal CAPES. Os descritores foram: paralisia facial e paralisia de Bell, combinados com reabilitação, terapia miofuncional e fonoaudiologia ou seus correlatos em inglês. Critérios de seleção: estudos disponíveis na íntegra, que abordaram a reabilitação fonoaudiológica em pessoas com PFP, publicados no período entre 1999 e 2019, em português brasileiro, inglês ou espanhol. Resultados: foram identificadas 650 publicações, mas apenas cinco artigos contemplaram os critérios de inclusão propostos. Dentre estes, a SciELO e PubMed obtiveram dois artigos incluídos cada. Além disso, a maioria foi publicada nos últimos dez anos e produzida no Brasil. O português brasileiro foi o idioma de publicação de três dos cinco artigos incluídos, não ocorrendo predominância de um nível de evidência específico. A descrição dos procedimentos utilizados não foi suficientemente detalhada nos estudos. Exercícios isotônicos e isométricos foram abordados mais frequentemente. A bandagem surgiu como recurso terapêutico em um estudo. Conclusão: embora haja um grande número de artigos relacionadas à PFP, apenas cinco estudos descreveram procedimentos fonoaudiológicos para pessoas com PFP, com nível de evidência baixo. Portanto, novos estudos abordando o tema são necessários.
\end{abstract}

Palavras-chave: Paralisia facial; Paralisia de Bell; Reabilitação; Terapia Miofuncional; Fonoaudiologia

\begin{abstract}
Purpose: identify and analyze the scientific production about the therapeutic strategies employed in the speech-language rehabilitation of people with peripheral facial paralysis. Research strategy: an integrative review was performed using the SciELO, PubMed, Web of Science, ScienceDirect and Portal CAPES databases. The descriptors were: facial paralysis and Bell's palsy, combined with rehabilitation, myofunctional therapy and Speech, Language and Hearing Sciences or their correlates in english. Selection criteria: studies available in their entirety, which addressed speech-language rehabilitation in people with peripheral facial paralysis, published between 1999 and 2019, in Brazilian Portuguese, english or spanish, were selected. Results: 650 publications were identified, but only five articles met the proposed inclusion criteria. Among these, SciELO and PubMed obtained two articles included each. In addition, most were published in the last ten years and produced in Brazil. Brazilian Portuguese was the language of publication of three of the five included, with no predominance of a specific level of evidence. The description of the procedures used was not sufficiently detailed in the studies. Isotonic and isometric exercises were approached more frequently. Bandage emerged as a therapeutic resource in one study. Conclusion: Although there are a large number of articles related to peripheral facial paralysis, only five studies described speechlanguage procedures for people with peripheral facial paralysis, with low level of evidence. Therefore, further studies addressing the topic are needed.
\end{abstract}

Keywords: Facial paralysis; Bell Palsy; Rehabilitation; Myofunctional Therapy; Speech, Language and Hearing Sciences

\footnotetext{
Trabalho realizado no Departamento de Formação Específica em Fonoaudiologia, Universidade Federal Fluminense - UFF - Nova Friburgo (RJ), Brasil.

${ }^{1}$ Instituto de Saúde de Nova Friburgo, Universidade Federal Fluminense - UFF - Nova Friburgo (RJ), Brasil.

${ }^{2}$ Departamento de Fonoaudiologia, Instituto de Ciências da Saúde - Universidade Federal da Bahia - UFBA - Salvador (BA), Brasil.

${ }^{3}$ Departamento de Formação Específica em Fonoaudiologia, Instituto de Saúde de Nova Friburgo - Universidade Federal Fluminense - UFF - Nova Friburgo (RJ), Brasil.

Conflito de interesses: Não.

Contribuição dos autores: MPD participou da idealização do estudo, coleta, análise e interpretação dos dados, redação e aprovação da versão final do artigo; MFFS participou da idealização do estudo, análise e interpretação dos dados, revisão e aprovação da versão final do artigo; SSB participou da idealização do estudo, supervisão da coleta, análise e interpretação dos dados, revisão e aprovação da versão final do artigo.
}

Financiamento: Nada a declarar.

Autor correspondente: Mabile Francine Ferreira Silva. E-mail: mabilef@hotmail.com

Recebido: Fevereiro 17, 2021; Aceito: Junho 28, 2021 


\section{INTRODUÇÃO}

A paralisia facial periférica (PFP) é uma condição neurológica decorrente da redução ou interrupção do transporte axonal ao nervo facial, que resulta em paralisia parcial ou completa dos músculos da mímica facial ${ }^{(1)}$. Diversas são as causas da PFP, dentre elas, as etiologias idiopática ou paralisia de Bell (PB), infecciosa, bacteriana sistêmica ou local, traumática, iatrogênica e metabólica ${ }^{(2)}$.

A PB é a causa de PFP mais encontrada na população. De acordo com a literatura internacional, sua incidência anual é de 11,5 a 40,2 casos por 100.000 habitantes. Essa condição pode afetar, ainda, um em cada 60 indivíduos ao longo da vida, sendo que há picos de incidência entre os 30 e 50 anos e aos 60 e 70 anos, nos quais não parece haver diferenciação na distribuição de frequência por gênero ou por hemiface comprometida ${ }^{(3)}$.

As sequelas da PFP causam importantes mudanças e dificuldades na vida das pessoas acometidas. O comprometimento da mímica e da expressão facial afeta os aspectos psíquico e social $^{(4,5)}$, com grande impacto sobre a aparência ${ }^{(6)}$, dificuldades na comunicação verbal e não verbal e, consequentemente, nas relações interpessoais ${ }^{(4,5)}$, além de ansiedade, estresse emocional e depressão $o^{(7)}$.

Quanto às demais funções orofaciais, são comumente encontradas na PFP alterações do tônus e da força da musculatura, provocando as mudanças na mímica e na expressão facial, além de ocasionar a produção inadequada dos fonemas bilabiais e labiodentais. Já as dificuldades na função mastigatória, são ocasionadas pela diminuição do tônus e da força do orbicular dos lábios e bucinador, resultantes da paralisia/paresia na hemiface afetada. Os indivíduos podem ter dificuldade para deglutir, em virtude da incompetência do vedamento labial, favorecendo o escape de alimentos ${ }^{(8-10)}$.

Devido ao amplo número de causas e aos impactos funcionais e estéticos dos quadros de PFP na vida das pessoas com esta condição, seu tratamento requer a atuação de uma equipe multidisciplinar, que pode ser composta por médico (otorrinolaringologista, oftalmologista e neurologista), fisioterapeuta, psicólogo e fonoaudiólogo ${ }^{(1,2)}$. A atuação em conjunto permite o cuidado integral da pessoa acometida e potencializa a evolução dos casos.

A atuação fonoaudiológica no processo de reabilitação da pessoa com PFP objetiva reduzir os efeitos da paralisia nas funções da mímica e da expressão facial, fala, mastigação, sucção e deglutição ${ }^{(11-16)}$. Na reabilitação miofuncional é fundamental manter a tonicidade muscular, além de readequar os aspectos funcionais e estéticos ${ }^{(10)}$.

No momento de definir as estratégias terapêuticas para reabilitação miofuncional de pessoas com PFP, é importante que o profissional fonoaudiólogo recorra à literatura científica para se atualizar e se preparar em relação à terapia fonoaudiológica. Sendo assim, é indispensável sintetizar os processos de reabilitação fonoaudiológica na PFP, com o intuito de facilitar a busca de novos programas e recursos terapêuticos pelos fonoaudiólogos, aperfeiçoando a prática clínica desses profissionais.

\section{OBJETIVOS}

Os objetivos deste estudo foram identificar e analisar a produção científica sobre as estratégias terapêuticas empregadas na reabilitação fonoaudiológica de pessoas com PFP.

\section{ESTRATÉGIA DE PESQUISA}

O desenho metodológico adotado para este estudo foi a revisão integrativa da literatura. Este método de pesquisa propicia a busca, a avaliação crítica e a síntese de evidências sobre um assunto específico e os resultados obtidos identificam lacunas, apontam a necessidade de novas pesquisas, bem como auxiliam os profissionais da saúde na escolha das condutas clínicas, ou na tomada de decisão ${ }^{(17)}$. O estudo foi delineado metodologicamente, a partir da questão norteadora: “Quais são os tipos de estratégias terapêuticas utilizadas por fonoaudiólogos na reabilitação miofuncional de pessoas com PFP?"

O levantamento das publicações foi realizado no período de julho a setembro de 2019, nas seguintes bases de dados eletrônicas: Scientific Eletronic Library Online (SciELO), PubMed, Web of Science - ISI, ScienceDirect e Portal de Periódicos da Coordenação de Aperfeiçoamento de Pessoal de Nível Superior (CAPES). Estas bases de dados foram escolhidas por serem as mais pesquisadas e por compilarem as principais revistas científicas do campo das ciências da saúde, além de terem acesso aberto para busca, ou via universidades públicas no Brasil.

Para a busca das publicações nas bases de dados, foram utilizados os seguintes descritores, identificados entre os Descritores em Ciências da Saúde (DeCS): "paralisia facial" e "paralisia de Bell" combinados com "reabilitação", "terapia miofuncional" e "fonoaudiologia", ou seus correlatos em inglês (facial paralysis, Bell paralisy, rehabilitation myofunctional therapy e speech, language and hearing sciences). As chaves de busca utilizadas foram: "paralisia facial" AND "reabilitação", "paralisia facial" AND "terapia miofuncional", "paralisia facial" AND "fonoaudiologia", bem como "paralisia de Bell" e as associações citadas anteriormente.

\section{CRITÉRIOS DE SELEÇÃo}

Para a seleção das publicações incluídas nesta revisão, foram definidos os seguintes critérios de inclusão: artigos científicos disponíveis na íntegra, que abordaram a reabilitação fonoaudiológica em pessoas com PFP, publicados no período entre 1999 e 2019, em português brasileiro, inglês ou espanhol. Os critérios de exclusão foram: publicações repetidas e estudos que não descreveram as estratégias terapêuticas utilizadas na reabilitação fonoaudiológica em PFP.

As etapas para a seleção dos estudos foram as seguintes: 1) pesquisa das publicações em cada base de dados definida; 2) sistematização de todos os estudos identificados no programa Microsoft Office Excel 2016, para controle dos pesquisadores; 3) pré-seleção desses estudos, conforme os critérios de inclusão estabelecidos, excluídos os artigos repetidos e não identificados; 4) seleção dos artigos que se relacionavam à pergunta norteadora, isto é, que abordavam a reabilitação 
fonoaudiológica de pessoas com PFP, por meio da leitura de título e resumo, quando disponíveis; 5) conferência de amostragem das publicações selecionadas em cada base de dados, realizada por um segundo avaliador; 6) leitura na íntegra e extração dos dados dos estudos selecionados, utilizando a ficha de coleta de dados desenvolvida para esta pesquisa; 7) definição dos artigos a serem incluídos na revisão, que descreviam as estratégias terapêuticas utilizadas; 8) classificação do nível de evidência científica dos estudos incluídos.

\section{ANÁLISE DE DADOS}

Os artigos selecionados foram analisados a partir dos dados extraídos por meio da ficha de coleta de dados, contemplando as seguintes variáveis de interesse: base de dados, ano de publicação, país de origem, idioma, título, objetivo do estudo, caracterização da amostra, procedimentos terapêuticos, resultados principais, conclusão, artigo incluído, ou não, e o nível de evidência. Optou-se por analisá-los, pois após a leitura de título e resumo, verificou-se que estes mencionavam algum procedimento terapêutico miofuncional voltado para reabilitação de pessoas com PFP. Tais dados também foram analisados em relação aos estudos incluídos. Foi realizada análise bibliométrica desses dados de identificação, sistematizando-os, a fim de compreender melhor as produções científicas sobre o tema investigado.

Quanto aos estudos incluídos, além de dados de identificação, foram sistematizadas informações sobre o objetivo do estudo, a caracterização da amostra envolvida, os procedimentos terapêuticos empregados, os principais resultados obtidos e sua conclusão. Adicionalmente, todos os trabalhos selecionados e incluídos nesta revisão foram analisados e classificados conforme os níveis de evidência científica estabelecidos pela American Speech-Language-Hearing Association (ASHA) em 2005(18) (Quadro 1). Os artigos não classificados segundo estes critérios foram qualificados com o seguinte termo: não se aplica (NSA).

\section{RESULTADOS}

$\mathrm{Na}$ etapa de busca nas bases de dados, foram encontradas 650 publicações. Após aplicação dos critérios de seleção e análise dos dados, 5 artigos foram incluídos nesta revisão (Figura 1).

Dentre as cinco bases de dados utilizadas, observou-se o predomínio de artigos selecionados na PubMED $(n=14)$, sendo que foram incluídos, nesta revisão integrativa, somente 2 estudos desta base e da SciELO. Na base ScienceDirect não foi selecionado nem incluído nenhum artigo.

Foram identificados 13 países diferentes dentro da amostra de estudos selecionados ( $\mathrm{n}=25$ ): Brasil, China, Coreia do Sul, Estados Unidos da América (EUA), Holanda, Índia, Irã, Itália, Japão, Malásia, Reino Unido, República Tcheca e Tunísia. Os países foram agrupados em seus respectivos continentes e foi possível observar um predomínio de publicações sobre o tema no continente americano $(40,0 \%)$. Dentre os artigos incluídos $(\mathrm{n}=5), 3$ foram produzidos no Brasil e os outros, na Holanda e no Japão.

Em relação ao idioma de publicação, das 25 pesquisas selecionadas, $76,0 \%(\mathrm{n}=19)$ foram publicadas na língua inglesa e as $24,0 \%(n=6)$ restantes na língua portuguesa (português brasileiro). Dos 5 estudos incluídos, 3 deles têm o português como idioma de publicação, e os outros, o inglês.

Para fins de descrição dos resultados obtidos nesta revisão quanto às estratégias terapêuticas empregadas na reabilitação de pessoas com PFP, o Quadro 2 apresenta os dados dos estudos incluídos, em relação ao objetivo do estudo, à amostra estudada, os procedimentos terapêuticos investigados, os resultados principais obtidos e as conclusões dos autores.

No Quadro 3, são apresentadas as estratégias terapêuticas descritas pelos autores em cada um dos estudos incluídos, abrangendo o programa terapêutico proposto (número e frequência das sessões terapêuticas).

Os artigos incluídos nesta revisão obtiveram as seguintes classificações, segundo a ASHA: 1b/3b (ensaios controlados randomizados de alta qualidade/estudos de coorte individual ou ensaios controlados randomizados de baixa qualidade); 4 (estudos de resultados clínicos); 6, 7 (opinião de especialistas sem avaliação crítica explícita) e NSA. Não foi observada, na classificação do nível de evidência, predominância de apenas uma classificação entre os estudos incluídos.

\section{DISCUSSÃO}

O presente estudo identificou e analisou publicações que abordavam os procedimentos terapêuticos utilizados por fonoaudiólogos na reabilitação miofuncional de pessoas com PFP. Conforme observado, apenas cinco dos 25 estudos selecionados se relacionavam à pergunta norteadora desta revisão, ou seja, descreviam estratégias terapêuticas atribuídas

Quadro 1. Níveis de evidência científica propostos pela American Speech-Language-Hearing Association

\begin{tabular}{|cl|}
\hline Níveis de evidência & \multicolumn{1}{c|}{ Tipos de estudo } \\
\hline $1 a$ & Revisão sistemática ou metanálise de alta qualidade de ensaios randomizados controlados \\
$1 b$ & Ensaios controlados randomizados de alta qualidade \\
$2 a$ & Revisão sistemática ou metanálise de alta qualidade de ensaios controlados não randomizados \\
$2 b$ & Ensaios controlados não randomizados de alta qualidade \\
$3 a$ & Revisão sistemática de estudos de coorte \\
$3 b$ & Estudos de coorte individual ou ensaios controlados randomizados de baixa qualidade \\
4 & Estudos de resultados clínicos \\
$5 a$ & Revisão sistemática de estudo de caso controle \\
$5 b$ & Estudo de caso controle individual \\
6 & Série de casos \\
7 & Opinião de especialistas sem avaliação crítica explícita \\
\hline
\end{tabular}




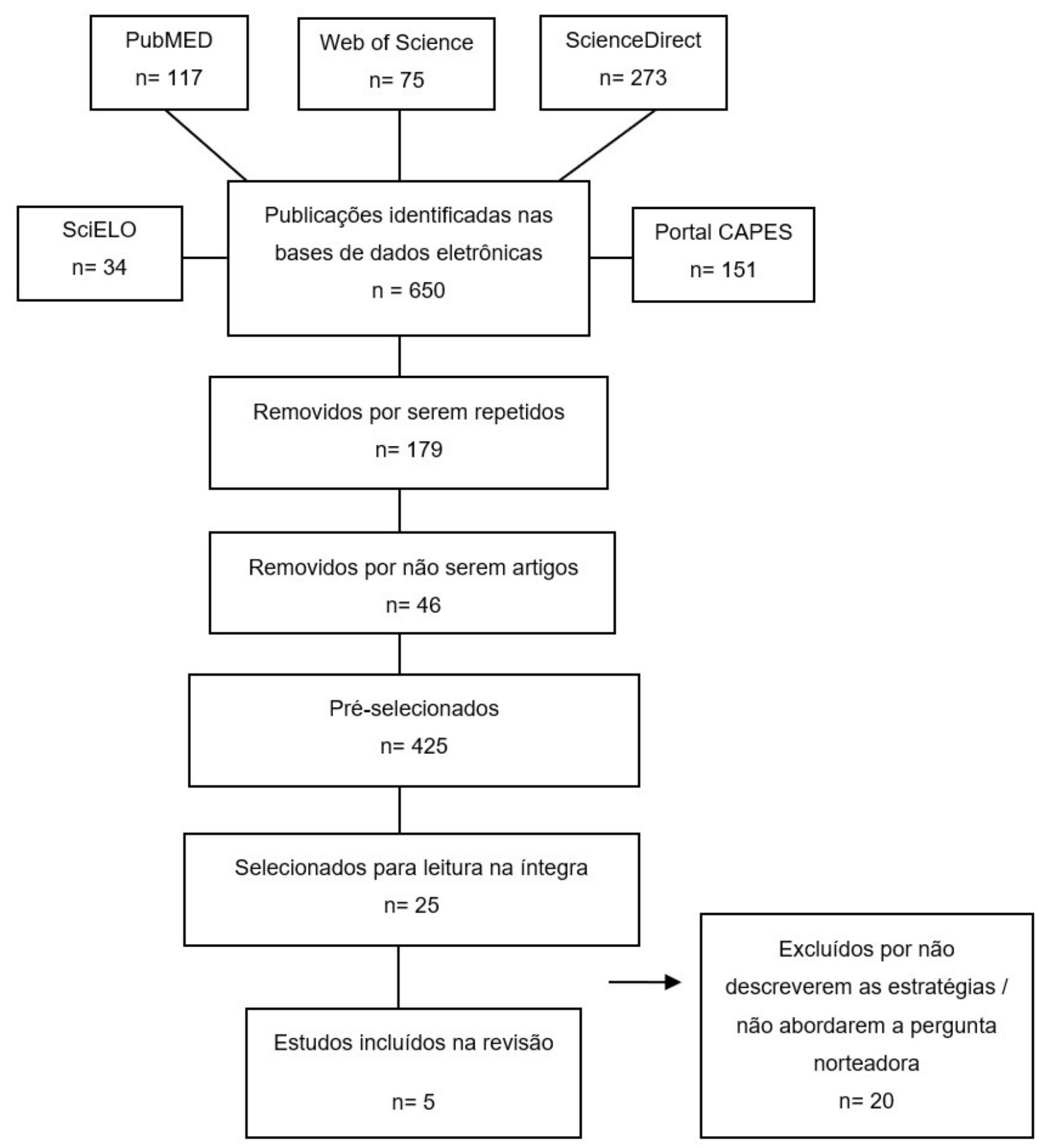

Figura 1. Fluxograma do processo de identificação, seleção e inclusão dos artigos

Legenda: $\mathrm{n}=$ número de artigos; CAPES = Coordenação de Aperfeiçoamento de Pessoal de Nível Superior

à Fonoaudiologia. As outras publicações selecionadas, embora tratassem de técnicas terapêuticas reabilitadoras da PFP, não eram práticas específicas da clínica fonoaudiológica, ou não descreviam de forma detalhada os procedimentos empregados. Portanto, são escassos na literatura estudos que apresentem ou que descrevam detalhadamente tais procedimentos.

A análise bibliométrica revelou que, na base SciELO, foi obtido o menor número de estudos identificados na etapa de busca, dentre todas as outras bases de dados eletrônicas, enquanto na PubMED obteve-se o maior número de artigos selecionados. Estas duas bases de dados possuem, juntas, quatro ${ }^{(19,21-23)}$ dos cinco estudos incluídos na amostra final (Figura 1). Por outro lado, na ScienceDirect, base em que houve o maior número de estudos identificados, nenhum deles abordava a intervenção fonoaudiológica na PFP de forma detalhada. Portanto, SciELO e PubMED parecem ser as bases de dados com melhor fonte de informação para o clínico interessado em reabilitar as funções miofuncionais na PFP. 


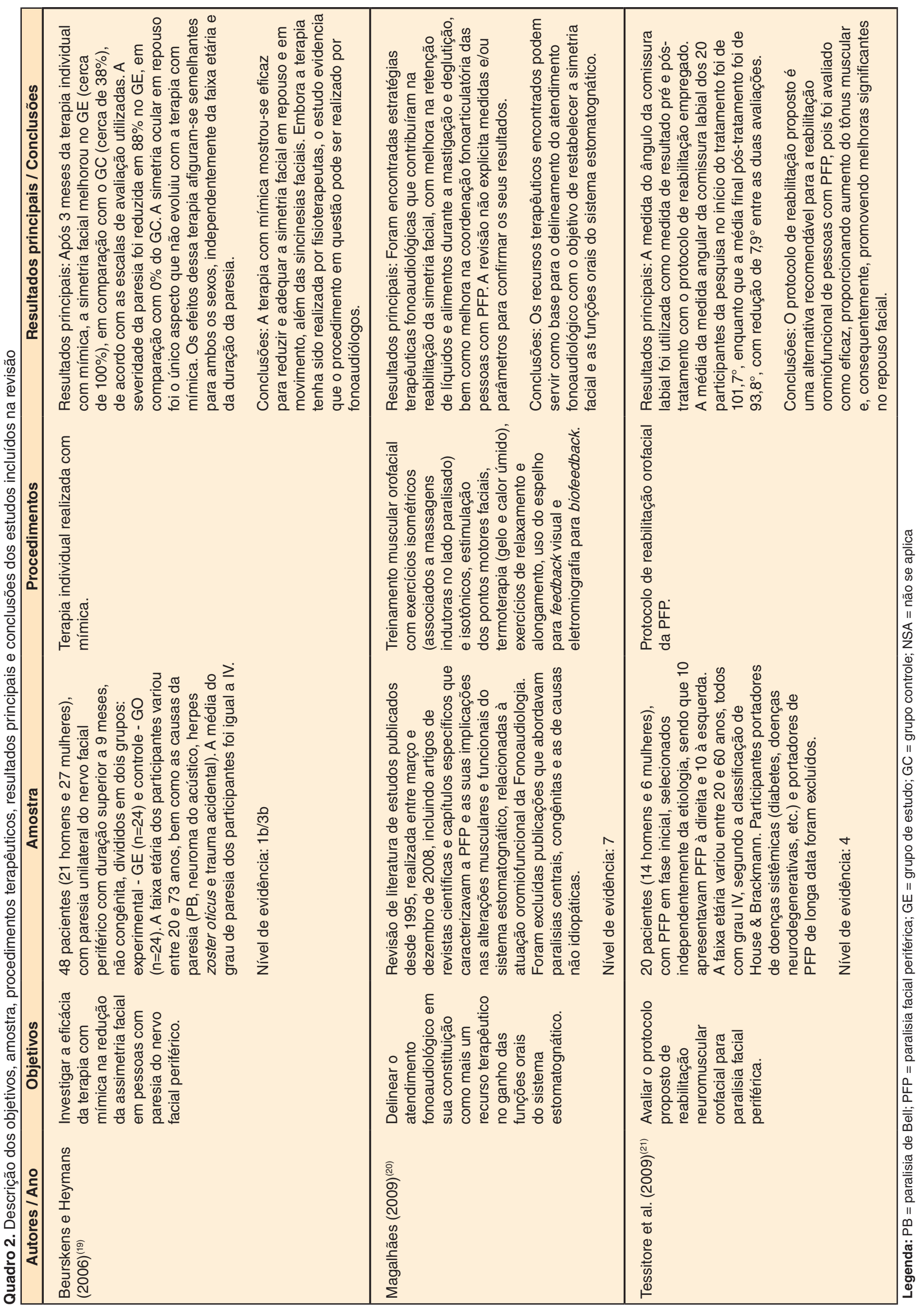




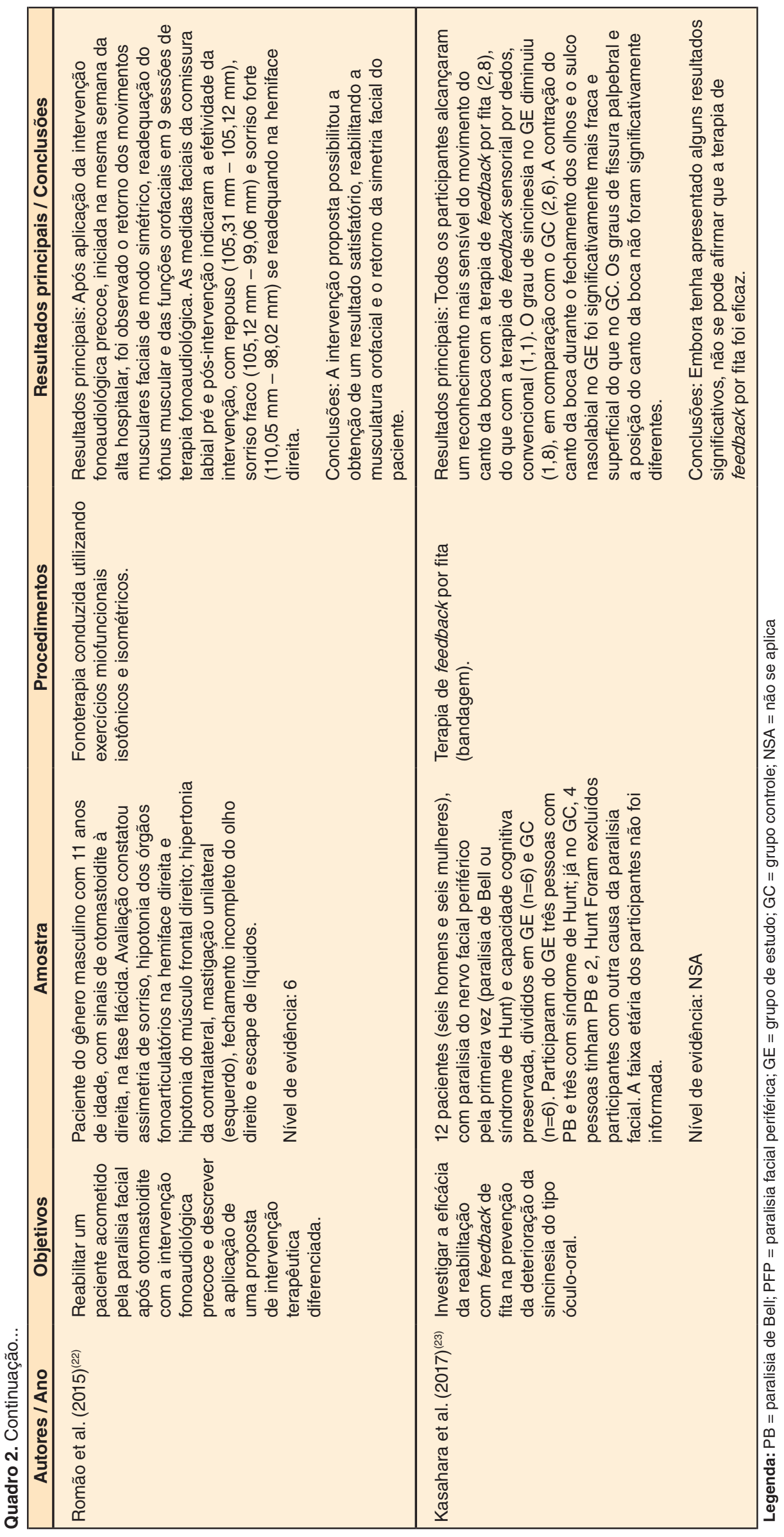




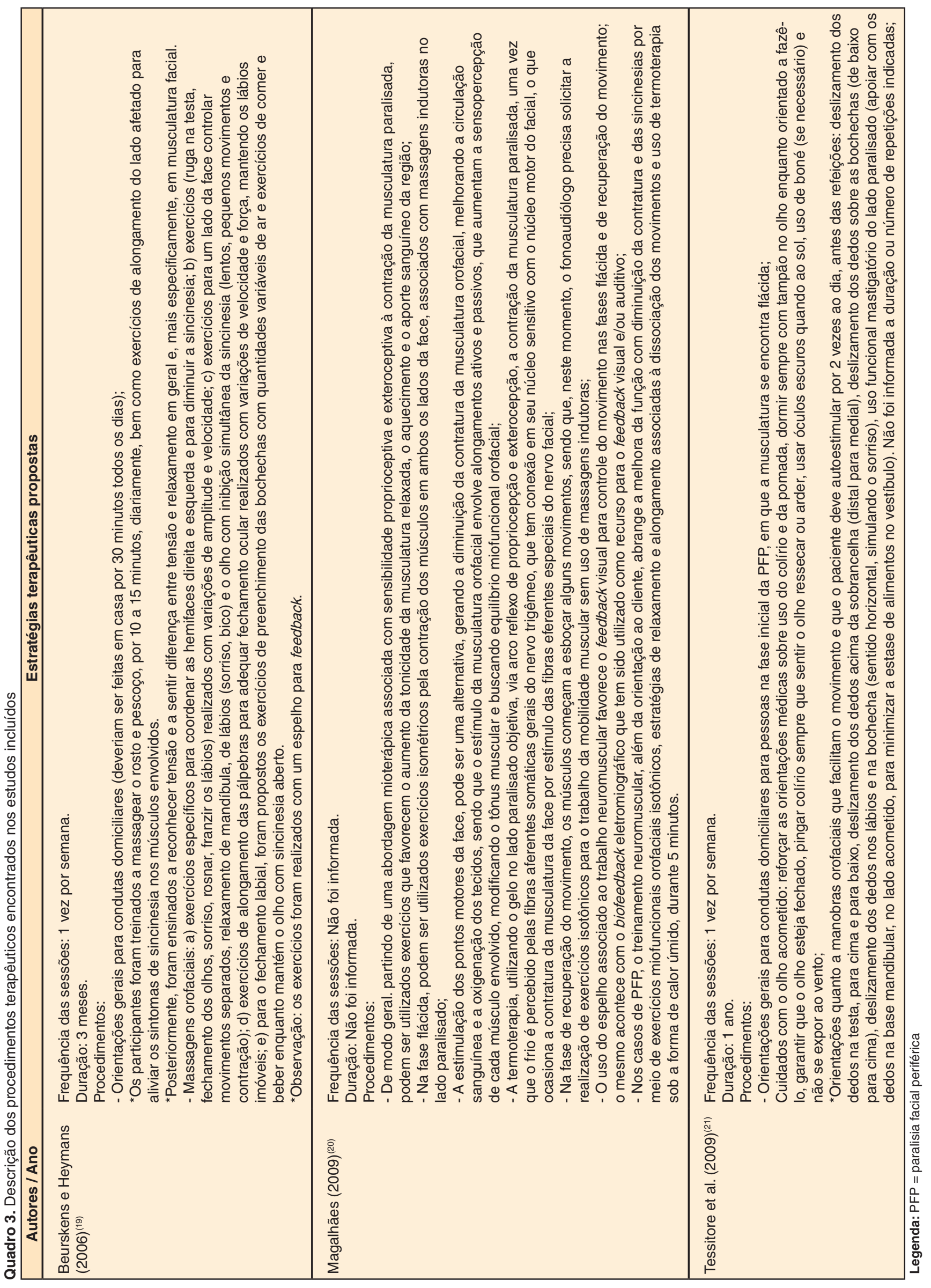




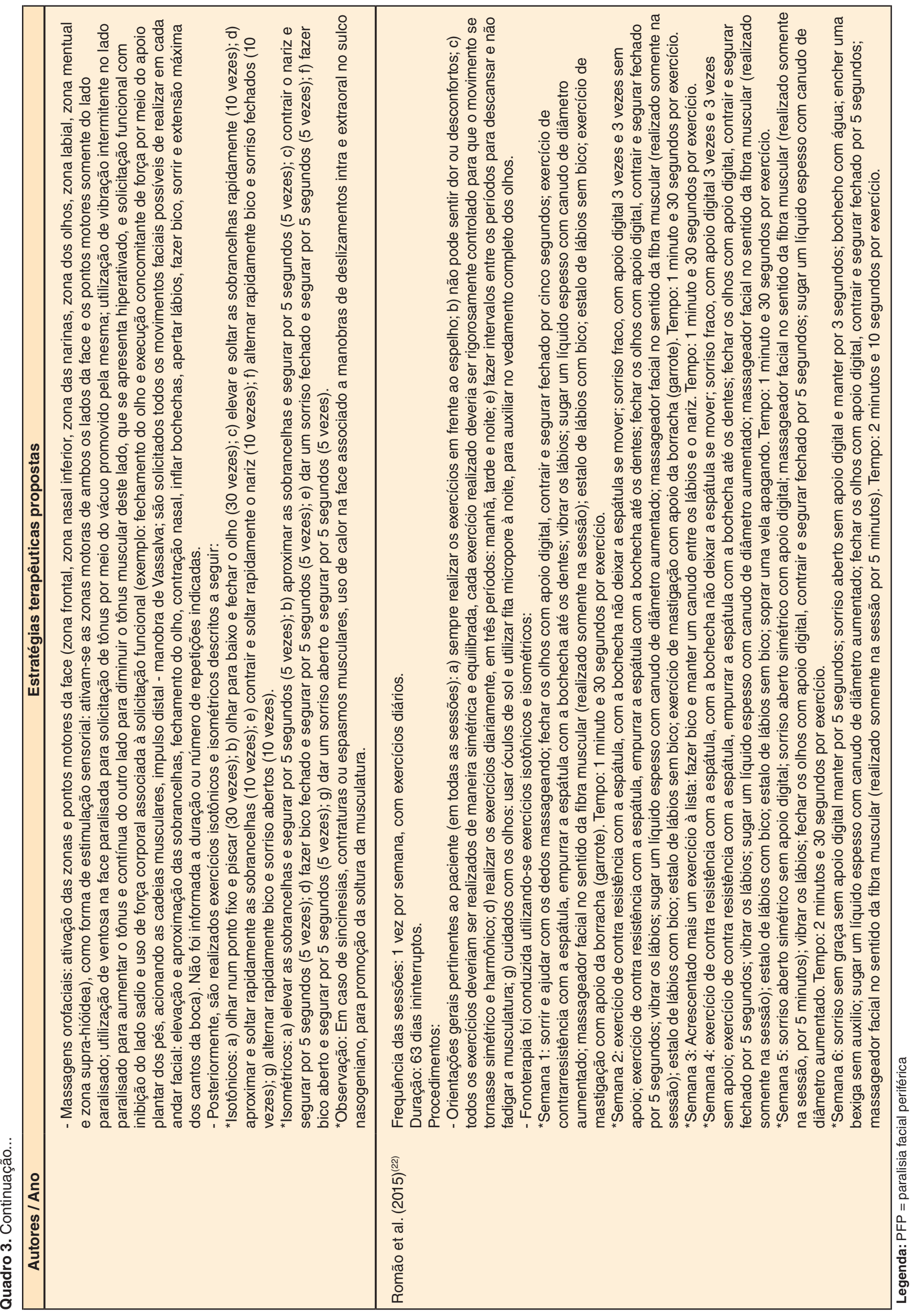




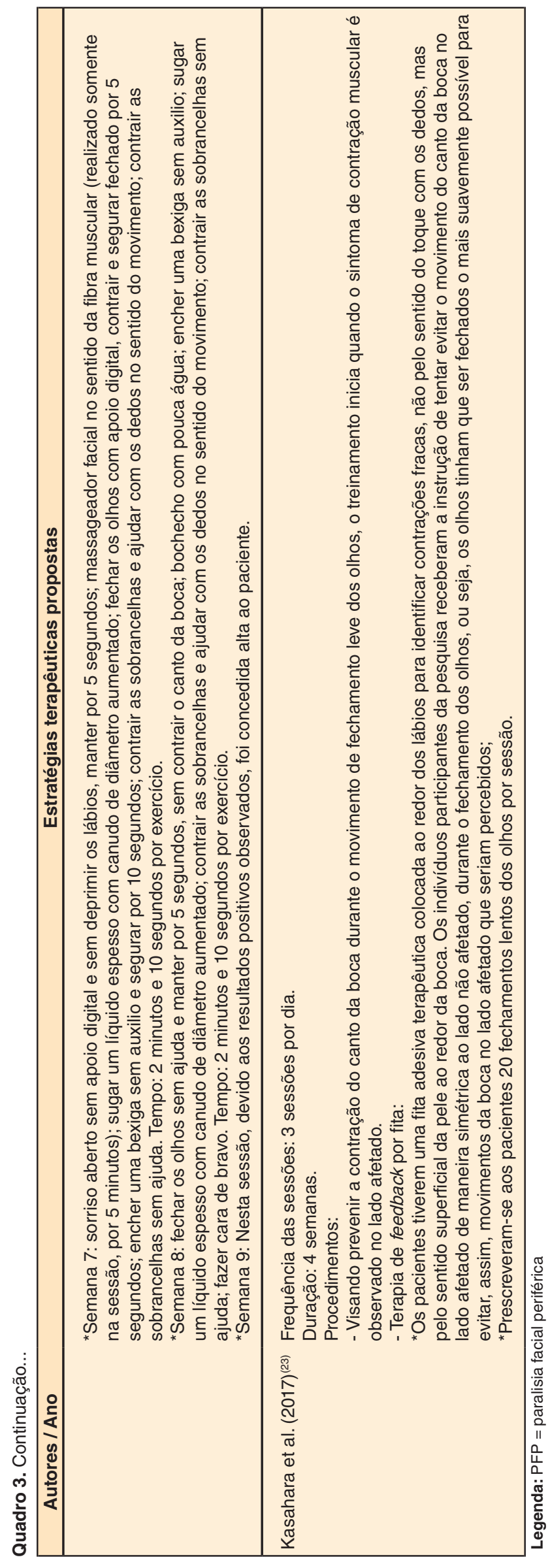


Quanto ao ano de publicação, observou-se que a maioria dos artigos foi publicada nos últimos dez anos, padrão também encontrado nos estudos incluídos ${ }^{(20-23)}$. Na literatura, foi encontrado estudo sobre terapia miofuncional orofacial, no qual foi possível observar padrão similar ${ }^{(24)}$. Possivelmente, tal padrão decorra do surgimento de novas estratégias e tecnologias para uso terapêutico, bem como do fortalecimento do movimento da prática baseada em evidências, o que pode explicar o aumento da produção científica na área fonoaudiológica sobre o tema. Não foram encontrados outros estudos abordando as possíveis causas do padrão citado anteriormente.

Em relação à distribuição das publicações por país de origem, o Brasil destacou-se na produção científica sobre intervenção fonoaudiológica na reabilitação miofuncional de pessoas acometidas por PFP no continente americano, cujas estratégias terapêuticas foram descritas em suas metodologias. Pode-se inferir portanto, que a Fonoaudiologia no Brasil oferece maior enfoque na reabilitação de pessoas com PFP do que em outros países, nos quais parece haver maior atuação da área de Fisioterapia.

$\mathrm{Na}$ Revista CEFAC foram encontrados dois ${ }^{(21,22)}$ dos estudos incluídos nesta revisão. Em um artigo recente de revisão de literatura $^{(25)}$, que analisou estudos clínicos de intervenção em motricidade orofacial em revistas científicas brasileiras de Fonoaudiologia, a Revista CEFAC foi o principal periódico no qual são publicados estudos na área de motricidade orofacial no país.

De modo geral, os objetivos dos estudos incluídos variaram, porém, em três deles, os resultados de propostas terapêuticas foram investigados ${ }^{(19,21,23)}$. Nos outros dois artigos, um de revisão de literatura ${ }^{(20)}$ e outro um estudo de caso $^{(22)}$, os objetivos traçados abordavam o delineamento da intervenção fonoaudiológica frente a casos de PFP, embora os níveis de evidência fossem distintos.

O perfil da amostra estudada se diferenciou entre os estudos incluídos. O tamanho da amostra variou de um a 48, totalizando 81 participantes em quatro ${ }^{(19,21-23)}$ estudos. A faixa etária dos participantes foi de 11 a 73 anos, com predomínio de homens. Quanto ao quadro clínico, 20 participantes foram classificados como grau IV da paralisia na escala de House-Brackmann. Nos demais, não foi especificado o grau, mas a fase da paralisia. Com relação à fase da PFP, 33 pacientes estavam na fase inicial e 48 com a afecção em período superior a nove meses. Na revisão de literatura incluída ${ }^{(20)}$, não foram especificadas as amostras de participantes dos seus estudos.

Dos quatro ${ }^{(19,21-23)}$ estudos que utilizaram medidas para analisar os resultados dos procedimentos terapêuticos propostos, dois usaram a medida do ângulo da comissura labial pré e pósintervenção, validando a efetividade das melhorias dos aspectos oromiofuncionais $^{(21,22)}$. Beurskens e Heymans ${ }^{(19)}$ utilizaram escalas de avaliação de aspectos orofaciais antes e após o tratamento, as quais confirmaram o restabelecimento da simetria facial e a diminuição da severidade da paresia. Neste estudo, a simetria ocular em repouso foi o único aspecto que se manteve após a intervenção proposta. Já no trabalho de Tessitore et al.(21), escalas de avaliação miofuncional e do grau de paralisia, além de documentações fotográficas, foram utilizadas para avaliar os resultados obtidos com a terapia ofertada.

No tocante às conclusões obtidas pelos autores dos estudos incluídos ${ }^{(19-23)}$ nesta revisão, os resultados favoráveis dos procedimentos terapêuticos fonoaudiológicos empregados, com aumento do tônus muscular e readequação da simetria facial, não foram pontos em comum. Desses, dois protocolos propostos e analisados foram considerados como recomendáveis na reabilitação miofuncional de pessoas com PFP, por seus autores ${ }^{(21,22)}$.

Dentre os procedimentos terapêuticos encontrados, evidenciase um padrão nas orientações aos pacientes, as quais deveriam ser realizadas em domicílio, de forma autônoma, em pelo menos três artigos ${ }^{(19,21,22)}$. As orientações abordavam o modo como os participantes deveriam realizar as massagens orofaciais, além de instruírem sobre a forma correta de realização dos exercícios.
Os estudos ${ }^{(19,21,22)}$ contemplaram, ainda, orientações pertinentes às pessoas com $\mathrm{PFP}$, como, por exemplo, cuidados com o olho acometido.

Embora os estudos incluídos tenham, de alguma forma, descrito as estratégias terapêuticas, constatou-se que a descrição não foi suficientemente detalhada em alguns casos, visto que informações importantes para a aplicação clínica correta e precisa das estratégias não foram fornecidas pelos autores. Destaca-se que em todas as publicações incluídas verificou-se falta de informações sobre as séries e/ou o tempo de realização de cada exercício. Uma possível explicação para este fato é de que os estudos não possuíam um protocolo de tratamento predefinido e que a terapia fonoaudiológica é individualizada, isto é, depende das queixas, dos resultados da avaliação fonaoudiológica e dos limites de cada indivíduo.

Os exercícios miofuncionais isotônicos e isométricos foram abordados como procedimentos terapêuticos, em três estudos ${ }^{(20-22)}$, sendo que o estudo de revisão ${ }^{(20)}$ apenas citou a possibilidade de uso desse tipo de exercício. O estudo de Kasahara et al. ${ }^{(23)}$ utilizou a bandagem como estratégia terapêutica, com um objetivo bem específico: investigar a efetividade desse recurso na prevenção da deterioração da sincinesia do tipo óculo-oral. A bandagem foi incorporada recentemente na prática fonoaudiológica, como recurso terapêutico, a fim de otimizar os resultados terapêuticos ${ }^{(26)}$. Esse recurso pode ser aplicado nas paralisias faciais, além de já ter demonstrado resultados efetivos como método terapêutico complementar no restabelecimento das alterações da motricidade orofacial $^{(27)}$.

Quanto ao esquema terapêutico em si, pôde-se observar que, em três estudos, a frequência das sessões sugerida foi de uma vez por semana ${ }^{(19,21,22)}$, sendo que no estudo de Romão et al. ${ }^{(22)}$ os exercícios também eram realizados diariamente. Outro estudo ${ }^{(23)}$ propôs um tratamento intensivo, com sessões três vezes ao dia e foi o único que utilizou bandagem nos procedimentos aplicados. No que tange à duração das intervenções propostas, diferenças foram observadas entre os estudos, variando de um mês a 12 meses.

Em relação à classificação do nível de evidência (Quadro 1), cinco artigos selecionados não se enquadraram nos critérios estabelecidos pela ASHA, pois o tipo de estudo não era contemplado nessa escala. Somente um artigo incluído não se enquadrou nos critérios de níveis de evidência ${ }^{(23)}$. No geral, os estudos selecionados e incluídos que puderam ser classificados apresentaram níveis de evidência baixos. Sendo assim, descrevendo, ou não, os procedimentos terapêuticos empregados no tratamento da PFP, as evidências científicas que embasam essa prática ainda não são robustas. Todavia, resultados positivos foram observados com essas intervenções no tratamento de pessoas com PFP.

Pondera-se que os baixos níveis de evidência encontrados limitam o desenvolvimento de uma prática fonoaudiológica baseada em evidências, visto que o clínico se depara com dificuldades de replicação das intervenções terapêuticas, devido à fragilidade dos critérios adotados para verificar a eficácia de tais procedimentos. Aos pesquisadores se faz necessário priorizar delineamentos de pesquisa capazes de encontrar evidência científica de maior nível e mais adequada ao método terapêutico investigado, bem como incluir a plena descrição da intervenção proposta.

Acredita-se que um limite desta revisão integrativa foi a não avaliação da qualidade metodológica dos estudos incluídos, para que a classificação dos níveis de evidência científica se desse de forma mais precisa. A incorporação de instrumentos formais de avaliação da qualidade das pesquisas em revisões deste tipo pode contribuir para a melhor compreensão do nível da produção de conhecimento sobre o tema, em termos qualitativos.

Diante do exposto, ressalta-se a necessidade de novas pesquisas, devido à escassez de estudos sobre a reabilitação fonoaudiológica em pessoas com PFP. Entretanto, é fundamental que as futuras investigações sejam realizadas com delineamento metodológico adequado, como ensaios clínicos controlados e randomizados, 
diminuindo, assim, os riscos de viés e aumentando a possibilidade de generalização dos resultados para uma tomada de decisão clínica mais assertiva e apropriada.

\section{CONCLUSÃO}

Há poucos estudos abordando protocolos ou terapêuticas fonoaudiológicas na PFP de forma detalhada, com forte nível de evidência, e que possibilitem a aplicabilidade clínica.

Quanto às estratégias terapêuticas encontradas, observou-se um padrão entre os estudos, em relação às orientações fornecidas pelos terapeutas, que consistiam no modo de realização dos exercícios em domicílio. Ademais, faltaram informações sobre o programa terapêutico em si, como, por exemplo, número de séries e/ou tempo de realização das intervenções. Exercício isotônicos e isométricos foram abordados na maioria dos procedimentos. A bandagem elástica surgiu como recurso terapêutico para potencializar a terapia fonoaudiológica.

\section{REFERÊNCIAS}

1. May M. Microanatomy and pathophysiology of the facial nerve. New York: Thieme; 1986. The facial nerve; p. 63-73.

2. Lazarini PR, Fouquet ML. Paralisia facial: avaliação, tratamento e reabilitação. São Paulo: Lovise; 2006.

3. De Diego JI, Prim MP, Madero R, Gavilán J. Seasonal patterns of idiopathic facial paralysis: a 16-year study. Otolaryngol Head Neck Surg. 1999;120(2):269-71. http://dx.doi.org/10.1016/S0194-5998(99)70418-3. PMid:9949364.

4. Silva MFF, Cunha MC, Lazarini PR, Fouquet ML. Conteúdos psíquicos e efeitos sociais associados à paralisia facial periférica: abordagem fonoaudiológica. Arq Int Otorrinolaringol. 2011;15(4):450-60. http:// dx.doi.org/10.1590/S1809-48722011000400008.

5. Silva MFF, Guedes ZCF, Cunha MC. Aspectos psicossociais associados à paralisia facial periférica na fase sequelar: estudo de caso clínico. Rev CEFAC. 2013;15(4):1025-31. http://dx.doi.org/10.1590/S151618462013000400033

6. Fernandes AMF, Lazarini PR. Anatomia do nervo facial. In: Lazarini PR, Fouquet ML, editores. Paralisia facial: avaliação, tratamento e reabilitação. São Paulo: Lovise; 2006. p. 1-10.

7. Silva MFF, Peres SV, Tessitore A, Paschoal JR, Cunha MC. Aplicação da escala psicossocial de aparência facial na avaliação da paralisia facial periférica: estudo piloto. Audiol Commun Res. 2016;21(0):e1618. http:// dx.doi.org/10.1590/2317-6431-2015-1618.

8. Finsterer J. Management of peripheral facial nerve palsy. Eur Arch Otorhinolaryngol. 2008;265(7):743-52. http://dx.doi.org/10.1007/ s00405-008-0646-4. PMid:18368417.

9. Mory MR, Tessitore A, Pfeilsticker LN, Couto EB Jr, Paschoal JR. Mastigação, deglutição e suas adaptações na paralisia facial periférica. Rev CEFAC. 2013;15(2):402-10. http://dx.doi.org/10.1590/S151618462012005000076 .

10. Silva MFF, Brito AF, Campos MF, Cunha MC. Atendimento multiprofissional da paralisia facial periférica: estudo de caso clínico. Distúrbios Comum. 2015;27(2):364-8.

11. Guedes ZCF. A atuação do fonoaudiólogo na equipe multidisciplinar de atendimento ao portador de paralisia facial periférica [tese]. São Paulo: Universidade Federal de São Paulo; 1994.
12. Goffi-Gomez MVS, Vasconcelos LGE, Moraes MFBB. Trabalho miofuncional na paralisia facial. Arq Fund Otorrinol. 1999;3(1):30-4.

13. Fouquet ML. Atuação fonoaudiológica nas paralisias faciais. In: Barros APB, Arakawa L, Tonini MD, Carvalho VA, organizador. Fonoaudiologia em cancerologia. São Paulo: Fundação Oncocentro; 2000. p. 99-104.

14. Altmann EBC, Vaz ACN. Paralisia facial: implicações da etiologia e das diferentes cirurgias. In: Comitê de Motricidade Orofacial da SBFa. Motricidade orofacial: como atuam os especialistas. São Paulo: Pulso; 2004. p. 187-98.

15. Bernardes DF, Gomez MV, Pirana S, Bento RF. Functional profile in patients with facial paralysis treated in a myofunctional approach. Pro Fono. 2004;16(2):151-8. PMid:15311739.

16. Calais LL, Gomez MV, Bento RF, Comerlatti LR. Avaliação funcional da mímica na paralisia facial central por acidente cerebrovascular. Pro Fono. 2005;17(2):213-22. http://dx.doi.org/10.1590/S0104-56872005000200010. PMid:16909531.

17. Mendes KDS, Silveira RCCP, Galvão CM. Revisão integrativa: método de pesquisa para a incorporação de evidências na saúde e na enfermagem. Texto Contexto Enferm. 2008;17(4):758-64. http://dx.doi.org/10.1590/ S0104-07072008000400018.

18. ASHA: American Speech and Hearing Association. An introduction to clinical trials [Internet]. 2005 [citado em 2019 Out 29]. Disponível em: https://leader.pubs.asha.org/doi/10.1044/leader.FTR3.10072005.6

19. Beurskens CHG, Heymans PG. Mime therapy improves facial symmetry in people with long-term facial nerve paresis: a randomised controlled trial. Aust J Physiother. 2006;52(3):177-83. http://dx.doi.org/10.1016/ S0004-9514(06)70026-5. PMid:16942452.

20. Magalhães HV Jr. Fonoterapia na paralisia facial periférica: uma abordagem miofuncional orofacial. RBPS. 2009;22(4):259-63. http:// dx.doi.org/10.5020/18061230.2009.p259.

21. Tessitore A, Paschoal JR, Pfeilsticker LN. Avaliação de um protocolo da reabilitação orofacial na paralisia facial periférica. Rev CEFAC. 2009;11(3, suppl 3):432-40. http://dx.doi.org/10.1590/S1516-18462009000700019.

22. Romão AM, Cabral C, Magni C. Intervenção fonoaudiológica precoce num paciente com paralisia facial após otomastoidite. Rev CEFAC. 2015;17(3):996-1003. http://dx.doi.org/10.1590/1982-021620159114.

23. Kasahara T, Ikeda S, Sugimoto A, Sugawara S, Koyama Y, Toyokura $\mathrm{M}$, et al. Efficacy of tape feedback therapy on synkinesis following severe peripheral facial nerve palsy. Tokai J Exp Clin Med. 2017;42(3):139-42. PMid:28871583.

24. Kayamori F, Bianchini EMG. Efeitos da terapia miofuncional orofacial em adultos quanto aos sintomas e parâmetros fisiológicos dos distúrbios respiratórios do sono: revisão sistemática. Rev CEFAC. 2017;19(6):86878. http://dx.doi.org/10.1590/1982-0216201719613317.

25. Tomaz-Morais J, Lima JAS, Luckwu-Lucena BT, Limeira RRT, Silva SM, Alves GÂS, et al. Estudos clínicos de intervenção em motricidade orofacial: análise metodológica de investigações brasileiras. Rev CEFAC. 2018;20(3):388-99. http://dx.doi.org/10.1590/1982-021620182032318.

26. Silva AP, Carvalho ARR, Sassi FC, Silva MAA. Os efeitos da bandagem elástica no músculo trapézio em adultos saudáveis. CoDAS. 2019;31(5):e20180077. http://dx.doi.org/10.1590/2317-1782/20192018077. PMid:31644716.

27. Silva AP, Escamez NES, Morini N Jr, Silva MAA. Método Therapy Taping®: bandagem elástica como recurso terapêutico na clínica Fonoaudiológica. Distúrbios da Comum. 2014;26(4):805-8. 